\title{
Electronic structure and resistivity of the double exchange model
}

\author{
DM Edwards†, ACM Green† and K Kuboł \\ $\dagger$ Department of Mathematics, Imperial College, London SW7 2BZ, UK \\ $\ddagger$ Institute of Physics, University of Tsukuba, Tsukuba, Ibaraki 305-8571, Japan
}

\begin{abstract}
The double exchange (DE) model with quantum local spins $S$ is studied; an equation of motion approach is used and decoupling approximations analogous to Hubbard's are made. Our approximate one-electron Green function $G$ is exact in the atomic limit of zero bandwidth for all $S$ and band filling $n$, and as $n \rightarrow 0$ reduces to a dynamical coherent potential approximation (CPA) due to Kubo; we regard our approximation as a many-body generalisation of Kubo's CPA. $G$ is calculated self-consistently for general $S$ in the paramagnetic state and for $S=1 / 2$ in a state of arbitrary magnetization. The electronic structure is investigated and four bands per spin are obtained centred on the atomic limit peaks of the spectral function. A resistivity formula appropriate to the model is derived from the Kubo formula and the paramagnetic state resistivity $\rho$ is calculated; insulating states are correctly obtained at $n=0$ and $n=1$ for strong Hund coupling. Our prediction for $\rho$ is much too small to be consistent with experiments on manganites so we agree with Millis et al that the bare DE model is inadequate. We show that the agreement with experiment obtained by Furukawa is due to his use of an unphysical density of states.
\end{abstract}

PACS numbers: 75.20.Hr, 72.90.+y, 71.28.+d

\section{Introduction}

Manganite compounds exhibiting colossal magnetoresistance (CMR) are of the form $\mathrm{La}_{1-x} \mathrm{D}_{x} \mathrm{MnO}_{3}$ with $\mathrm{D}$ divalent, e.g. Ca, Sr, Ba. As the doping $x$ and temperature $T$ are varied a rich variety of phases are observed, as discussed by Ramirez [四]. Recently there has been a lot of interest in these compounds with $x \gtrsim 0.15$ owing to their interesting magnetotransport properties in this regime: as $T$ is decreased they undergo a transition to ferromagnetic order, and near the Curie temperature $T_{\mathrm{C}}$ the $T$-dependence of the resistivity $\rho$ changes from insulating $\left(\partial \rho / \partial T<0\right.$ for $\left.T>T_{\mathrm{C}}\right)$ to metallic $(\partial \rho / \partial T>0$ for $\left.T<T_{\mathrm{C}}\right)$, with a strong peak in $\rho$ at the crossover. The application of a strong $(\sim 5 \mathrm{~T})$ magnetic field substantially reduces this peak in $\rho$ and shifts it to higher temperature, giving rise to a very large negative magnetoresistance. The physical processes causing this behaviour have been the subject of much discussion. 
The simplest model proposed for the CMR compounds - the one that we will study in this paper - is Zener's [2] double exchange (DE) model with Hamiltonian

$$
H=\sum_{i j \sigma} t_{i j} c_{i \sigma}^{\dagger} c_{j \sigma}-J \sum_{i} \vec{S}_{i} \cdot \vec{\sigma}_{i}=H_{0}+H_{1}
$$

Here $i$ and $j$ refer to sites of the (approximately) simple cubic lattice of Mn atoms, $c_{j \sigma}\left(c_{i \sigma}^{\dagger}\right)$ is a $\sigma$-spin conduction electron annihilation (creation) operator, $\vec{S}_{i}$ is a local spin operator, $\vec{\sigma}_{i}$ is a conduction electron spin operator, $t_{i j}$ is the hopping integral with discrete Fourier transform $t_{\vec{k}}$, and $J>0$ is the Hund's rule coupling constant. The number of conduction electrons per atom $n$ is assumed to be given by $n=1-x$. Physically, the relevant electrons are those coming from the Mn atoms' $3 \mathrm{~d}$ shells, which contain four electrons per site in the undoped compounds and are split by the cubic crystal field into triply degenerate $t_{2 g}$ levels and higher energy doubly degenerate $e_{g}$ levels. Strong Hund coupling attempts to align all electron spins on a site, so the $t_{2 g}$ electrons are treated as localized $S=3 / 2$ spins while the conduction band is formed from the $e_{g}$ states. The main physical effects neglected by $H$ are the double degeneracy of the $e_{g}$ conduction band, impurity atom (D) disorder scattering, and coupling to the lattice degrees of freedom.

Furukawa [3] has studied the infinite dimensional limit of $H$ for $S=\infty$ using dynamical mean field theory, and has concluded that the DE model's predictions for $\rho$ in the paramagnetic state are compatible with experiment. Millis et al [4 :5] however have claimed that $\rho$ predicted by the DE model is much smaller than that measured, and that to get agreement with experiment dynamical Jahn-Teller phonon coupling must be included in $H$. Experiments show that phonon coupling is important, with for example a large shift in $T_{\mathrm{C}}$ observed upon replacing some of the $\mathrm{O}$ atoms with a different isotope [6], so Furukawa's claim is puzzling. In this paper we will study the one-electron local Green function $G$ and calculate $\rho$ in an attempt to reconcile the results of Millis and Furukawa. This work has been briefly summarised elsewhere [7, 8] and is discussed in more detail by Green [9].

Our starting point is Kubo's calculation based on a dynamical coherent potential approximation (CPA). For finite local spins $S$ dynamical scattering processes may occur in which local spins and conduction electrons exchange angular momentum, whereas in the classical $S \rightarrow \infty$ limit taken by most authors the local spins are rigid and $H$ is a one-electron Hamiltonian with spin dependent diagonal disorder. Kubo's CPA is an extension of the familiar alloy CPA which takes these dynamical processes into account in a local approximation. Since it is a one-electron theory Kubo's approximation is only valid in the low-density $n \rightarrow 0$ limit, but in this limit the behaviour of the spectral function is qualitatively correct with bands with the correct weights forming about the two atomic limit $\left(t_{i j} \rightarrow 0\right)$ peaks as the hopping $t_{i j}$ is switched on. If $J \gg t_{i j}$ double occupation of a site is forbidden so that at half-filling $(n=1)$ the system should be a Mott insulator. This is not the case in Kubo's CPA where the Fermi level lies within the lower band. The correct behaviour will be obtained in an approximation which becomes exact in the atomic limit for all filling, so we are looking for a many-body extension of Kubo's CPA, valid for all $n$, which reduces to Kubo's CPA as $n \rightarrow 0$ and to the correct 
atomic limit as $t_{i j} \rightarrow 0$, for all $n$.

It is difficult to extend the usual CPA method to the many-body case. Instead we return to the original approach of Hubbard [10] in which he applied the equation of motion method to calculate the one-electron local Green function $G$ for the Hubbard model. His decoupling approximation was motivated by the alloy analogy in which electrons of one spin are considered as frozen on atomic sites. Hubbard's 'scattering correction' is equivalent to a CPA treatment of the alloy analogy [1] and his 'resonance broadening correction' was an attempt to restore some dynamics to the frozen electrons. The idea of $\downarrow$ spins being frozen in the calculation of the $\uparrow$ spin Green function $G_{\uparrow}$ is introduced in the equation of motion method by neglecting commutators of the kinetic part of the Hamiltonian $H_{0}$ with $\downarrow$-spin occupation numbers.

In the derivation of our approximation to $G$ for the DE model we will make approximations analogous to those used by Hubbard to obtain the scattering correction; terms corresponding to his resonance broadening correction will be neglected. Our method represents a considerable extension of Hubbard's owing to the more complicated form of the interaction term of the DE model, which for instance allows electrons to change spin via exchange of angular momentum with the local spins. This effect couples the equations for $G_{\uparrow}$ and $G_{\downarrow}$ which may perhaps be regarded as including some resonance broadening effects. Our choice of approximations will be guided by the requirement that we recover Kubo's CPA as $n \rightarrow 0$ and the correct atomic limit as $t_{i j} \rightarrow 0$. Owing to the spin symmetry of $H$ we only need to derive an equation for $G_{\uparrow}$, and the equation for $G_{\downarrow}$ follows immediately.

We calculate the atomic limit Green function $g$ exactly in section 2 and derive our many-body CPA equation for the Green function $G$ in section 3 . The CPA spectral function is studied in section 4 . In the limit of infinite $J$ and $n \rightarrow 1$, Kubo [12 has introduced a CPA treatment of holes and we compare our results with this in section section 5. A formula for the resistivity $\rho$ of the zero field paramagnetic state is derived in section 6 and in section $0 \rho$ is calculated for various approximations to the density of states (DOS). A summary is given in section 8 .

\section{Atomic limit}

Since we require our approximation for $G$ to be exact in the atomic limit we must first derive $G$ in this limit. We define the retarded Green function for operators $A$ and $B$ with no explicit time-dependence by $\langle\langle A ; B\rangle\rangle_{t}=-\mathrm{i} \theta^{+}(t)\langle\{A(t), B\}\rangle$ and its Fourier transform by $\langle\langle A ; B\rangle\rangle_{\epsilon}=\int_{-\infty}^{\infty} \mathrm{d} t \mathrm{e}^{\mathrm{i} \epsilon t}\langle\langle A ; B\rangle\rangle_{t}$. Here $\theta^{+}(x)=1$ for $x>0$ and 0 otherwise, $\epsilon$ is restricted to the upper half of the complex plane, and [,] and $\{$,$\} are the$ commutator and anticommutator respectively. The equation of motion for the latter Green function is

$$
\epsilon\langle\langle A ; B\rangle\rangle_{\epsilon}=\langle\{A, B\}\rangle+\langle\langle[A, H] ; B\rangle\rangle_{\epsilon} .
$$

The $\sigma$-spin one-electron Green function is given by $G_{\sigma}^{i j}(\epsilon)=\left\langle\left\langle c_{i \sigma} ; c_{j \sigma}^{\dagger}\right\rangle\right\rangle_{\epsilon}$, and we define $G_{\sigma}=G_{\sigma}^{i i}$ and $G_{\vec{k} \sigma}=N^{-1} \sum_{\vec{R}_{i j}} \exp \left(\mathrm{i} \vec{k} \cdot \vec{R}_{i j}\right) G_{\sigma}^{i j}$ where $\vec{R}_{i j}$ is the vector from the $j^{\text {th }}$ to 
the $i^{\text {th }}$ lattice site and $N$ is the number of sites. The occupation number operators are

$n_{i \sigma}=c_{i \sigma}^{\dagger} c_{i \sigma}$ and we introduce the notation $n_{i \sigma}^{\alpha}$, with $\alpha= \pm$, such that $n_{i \sigma}^{+}=n_{i \sigma}$ and $n_{i \sigma}^{-}=1-n_{i \sigma}$. We also define the total occupation number $n_{i}=n_{i \uparrow}+n_{i \downarrow}$ and $n_{i}^{+}=n_{i}$, $n_{i}^{-}=1-n_{i}$. We denote the Green function $G_{\sigma}$ in the atomic limit $t_{i j}=0$ by $g_{\sigma}$.

In the atomic limit the eigenstates and energy eigenvalues of $H$ are completely defined in terms of those of the single-site Hamiltonian. In order of increasing energy these consist of $2(S+1 / 2)+1=2 S+2$ singly occupied states with electron spin and local spin parallel having energy $-J S / 2,2 S+1$ unoccupied states and $2 S+1$ doubly occupied states with energy 0 , and $2(S-1 / 2)+1=2 S$ singly occupied states with electron spin and local spin antiparallel having energy $J(S+1) / 2 . g_{\uparrow}$ is easily obtained using the equation of motion method. We define $g_{\uparrow}^{\alpha}=\left\langle\left\langle n^{\alpha} c_{\uparrow} ; c_{\uparrow}^{\dagger}\right\rangle\right\rangle, s_{\uparrow}^{\alpha}=\left\langle\left\langle n^{\alpha} S^{z} c_{\uparrow} ; c_{\uparrow}^{\dagger}\right\rangle\right\rangle$, and $t_{\uparrow}^{\alpha}=\left\langle\left\langle n^{\alpha} S^{-} c_{\downarrow} ; c_{\uparrow}^{\dagger}\right\rangle\right\rangle$, dropping site indices since the site referred to is always the same. $S^{ \pm}$and $\sigma^{ \pm}$are the raising/lowering operators for the local spins and conduction electrons respectively. We start with $g^{\alpha}$ and write equations of motion for undetermined Green functions until the system of equations closes, obtaining

$\epsilon g_{\uparrow}^{\alpha}(\epsilon)=\left\langle n_{\downarrow}^{\alpha}\right\rangle-\frac{J}{2}\left[s_{\uparrow}^{\alpha}(\epsilon)+t_{\uparrow}^{\alpha}(\epsilon)\right]$
$\epsilon\left[s_{\uparrow}^{\alpha}(\epsilon)+t_{\uparrow}^{\alpha}(\epsilon)\right]=\left\langle S^{z} n_{\downarrow}^{\alpha}-\alpha S^{-} \sigma^{+}\right\rangle-\frac{J \alpha}{2}\left[s_{\uparrow}^{\alpha}(\epsilon)+t_{\uparrow}^{\alpha}(\epsilon)\right]-\frac{J S(S+1)}{2} g_{\uparrow}^{\alpha}(\epsilon)$.

Now $g_{\uparrow}=\sum_{\alpha= \pm} g_{\uparrow}^{\alpha}$, and solving (3a) and $(3 b)$ for $g^{\alpha}$ we obtain

$$
\begin{aligned}
g_{\uparrow}(\epsilon)=\frac{1}{2 S+1}\left[\frac{\left\langle\left(S+S^{z}\right) n_{\downarrow}-S^{-} \sigma^{+}\right\rangle}{\epsilon+J(S+1) / 2}+\frac{\left\langle\left(S-S^{z}\right)\left(1-n_{\downarrow}\right)-S^{-} \sigma^{+}\right\rangle}{\epsilon-J(S+1) / 2}\right. \\
\left.+\frac{\left\langle\left(S+1+S^{z}\right)\left(1-n_{\downarrow}\right)+S^{-} \sigma^{+}\right\rangle}{\epsilon+J S / 2}+\frac{\left\langle\left(S+1-S^{z}\right) n_{\downarrow}+S^{-} \sigma^{+}\right\rangle}{\epsilon-J S / 2}\right] .
\end{aligned}
$$

It should be noted that for finite $S$ there are four peaks in the spectral function at energies $\pm J(S+1) / 2$ and $\pm J S / 2$; for $S \rightarrow \infty$ the upper and lower pairs of peaks merge leaving the familiar double-peaked spectral function. To obtain $g_{\downarrow}(\epsilon)$ from (雨) we make the changes $n_{\sigma} \mapsto n_{-\sigma}, S^{z} \mapsto-S^{z}, S^{ \pm} \mapsto S^{\mp}, \sigma^{ \pm} \mapsto \sigma^{\mp}$. The weight summed over spin in the lowest band is $(n S-2\langle\vec{S} \cdot \vec{\sigma}\rangle) /(2 S+1)$, and since $\langle\vec{S} \cdot \vec{\sigma}\rangle \rightarrow n S / 2$ as the temperature $T \rightarrow 0$ for sufficiently large $J$ the peak at $-J(S+1) / 2$ will have very little weight at reasonable temperatures. Hence it will often be a good approximation to neglect the lowest band. The total weight summed over spin in the lowest two bands is $(2 S+2-n) /(2 S+1)$, so it is clear that a $t_{i j} \neq 0$ theory that becomes exact as $t_{i j} \rightarrow 0$ will give an insulator at half-filling.

\section{CPA Green function}

Our general strategy in this section follows that of Hubbard - we will retain occupation numbers as operators rather than replacing them with their averages, but their commutators with $H_{0}$ will be neglected where appropriate, corresponding to the frozen electron approximation of the alloy analogy. Terms which are not multiplied by $t_{i j}$ must be treated exactly in order to obtain the correct atomic limit. 
We split $G_{\uparrow}^{i j}$ into two components, $G_{\uparrow}^{i j}=\sum_{\alpha= \pm} G_{\uparrow}^{i j \alpha}$ where $G_{\uparrow}^{i j \alpha}(\epsilon)=\left\langle\left\langle n_{i}^{\alpha} c_{i \uparrow} ; c_{j \uparrow}^{\dagger}\right\rangle\right\rangle_{\epsilon}$, corresponding to propagation through singly $(\alpha=-)$ and doubly $(\alpha=+)$ occupied sites. Neglecting $\left[n_{i \downarrow}^{\alpha}, H_{0}\right]$ the equation of motion for each component is then given by

$$
\begin{gathered}
\epsilon G_{\uparrow}^{i j \alpha}(\epsilon) \approx\left\langle n_{i \downarrow}^{\alpha}\right\rangle\left(\delta_{i j}+\sum_{k} t_{i k} G_{\uparrow}^{k j}(\epsilon)\right)+\sum_{k} t_{i k}\left\langle\left\langle\delta n_{i \downarrow}^{\alpha} c_{k \uparrow} ; c_{j \uparrow}^{\dagger}\right\rangle\right\rangle_{\epsilon} \\
-\frac{J}{2}\left(S_{\uparrow}^{i j \alpha}(\epsilon)+T_{\uparrow}^{i j \alpha}(\epsilon)\right)
\end{gathered}
$$

where we have introduced the Green functions $S_{\uparrow}^{i j \alpha}(\epsilon)=\left\langle\left\langle n_{i}^{\alpha} S_{i}^{z} c_{i \uparrow} ; c_{j \uparrow}^{\dagger}\right\rangle\right\rangle_{\epsilon}$ and $T_{\uparrow}^{i j \alpha}(\epsilon)=$ $\left\langle\left\langle n_{i}^{\alpha} S_{i}^{-} c_{i \downarrow} ; c_{j \uparrow}^{\dagger}\right\rangle\right\rangle_{\epsilon}$ and the notation $\delta A=A-\langle A\rangle$ for any operator $A$. The second term on the right-hand side of (5) is Hubbard's scattering correction in which the deviation of $n_{i \downarrow}^{\alpha}$ from its average is accounted for. The last term of (5) is more complicated than in the case of the Hubbard model, containing the as yet undetermined Green functions $S_{\uparrow}$ and $T_{\uparrow}$. These correspond respectively to propagation of the electron as an $\uparrow$-spin and, following spin-flip scattering from a local spin, as a $\downarrow$-spin; the presence of $T_{\uparrow}$ will couple the equations for $G_{\uparrow}$ and $G_{\downarrow}$.

We first treat the scattering correction, splitting the relevant Green function into two components, $\left\langle\left\langle\delta n_{i \downarrow}^{\alpha} c_{k \uparrow} ; c_{j \uparrow}^{\dagger}\right\rangle\right\rangle_{\epsilon}=\sum_{\beta= \pm}\left\langle\left\langle\delta n_{i \downarrow}^{\alpha} n_{k}^{\beta} c_{k \uparrow} ; c_{j \uparrow}^{\dagger}\right\rangle\right\rangle_{\epsilon}$. It is assumed that $t_{i i}=0$, so from (5) it may be seen that this Green function is needed only for $i \neq k$. The equations of motion are, for $i \neq k$,

$$
\begin{gathered}
\epsilon\left\langle\left\langle\delta n_{i \downarrow}^{\alpha} n_{k}^{\beta} c_{k \uparrow} ; c_{j \uparrow}^{\dagger}\right\rangle\right\rangle_{\epsilon} \approx\left\langle\delta n_{i \downarrow}^{\alpha} \delta n_{k \downarrow}^{\beta}\right\rangle \delta_{j k}+\sum_{l} t_{k l}\left\langle\left\langle\delta n_{i \downarrow}^{\alpha} n_{k \downarrow}^{\beta} c_{l \uparrow} ; c_{j \uparrow}^{\dagger}\right\rangle\right\rangle_{\epsilon} \\
-\frac{J}{2}\left\langle\left\langle\delta n_{i \downarrow}^{\alpha} n_{k}^{\beta}\left(S_{k}^{z} c_{k \uparrow}+S_{k}^{-} c_{k \downarrow}\right) ; c_{j \uparrow}^{\dagger}\right\rangle\right\rangle_{\epsilon}
\end{gathered}
$$

where $\left[n_{k \downarrow}^{\beta}, H_{0}\right]$ and all commutators involving $\delta n_{i \downarrow}^{\alpha}$ have been neglected. This is consistent with the strategy stated above. As a further approximation the first term on the right-hand side of (6), a two-site correlation function, is dropped and we set $n_{k \downarrow}^{\beta} \approx\left\langle n_{k \downarrow}^{\beta}\right\rangle$ in the second term, which corresponds to neglecting a second scattering correction.

The system of equations for the scattering correction is now closed apart from the last Green function in (6). In the equation of motion for this term we use the fact that

$$
n_{k}^{\beta}\left(S_{k}^{z} c_{k \uparrow}+S_{k}^{-} c_{k \downarrow}\right)= \begin{cases}\left(S_{k}^{z} n_{k \downarrow}^{\beta}-\beta S_{k}^{-} \sigma_{k}^{+}\right) c_{k \uparrow} & \text { for } \beta=+ \\ c_{k \uparrow}\left(S_{k}^{z} n_{k \downarrow}^{\beta}-\beta S_{k}^{-} \sigma_{k}^{+}\right) & \text {for } \beta=-\end{cases}
$$

and replace $S_{k}^{z} n_{k \downarrow}^{\beta}-\beta S_{k}^{-} \sigma_{k}^{+}$by its average in the Green function coming from $H_{0}$. Neglecting two-site correlation functions and commutators of $\delta n_{i \downarrow}^{\alpha}$ we obtain the equation of motion, for $i \neq k$,

$$
\begin{gathered}
\epsilon\left\langle\left\langle\delta n_{i \downarrow}^{\alpha} n_{k}^{\beta}\left(S_{k}^{z} c_{k \uparrow}+S_{k}^{-} c_{k \downarrow}\right) ; c_{j \uparrow}^{\dagger}\right\rangle\right\rangle_{\epsilon} \approx\left\langle S_{k}^{z} n_{k \downarrow}^{\beta}-\beta S_{k}^{-} \sigma_{k}^{+}\right\rangle \sum_{l} t_{k l}\left\langle\left\langle\delta n_{i \downarrow}^{\alpha} c_{l \uparrow} ; c_{j \uparrow}^{\dagger}\right\rangle\right\rangle_{\epsilon} \\
-\frac{J \beta}{2}\left\langle\left\langle\delta n_{i \downarrow}^{\alpha} n_{k}^{\beta}\left(S_{k}^{z} c_{k \uparrow}+S_{k}^{-} c_{k \downarrow}\right) ; c_{j \uparrow}^{\dagger}\right\rangle\right\rangle_{\epsilon} \\
-\frac{J S(S+1)}{2}\left\langle\left\langle\delta n_{i \downarrow}^{\alpha} n_{k}^{\beta} c_{k \uparrow} ; c_{j \uparrow}^{\dagger}\right\rangle\right\rangle_{\epsilon},
\end{gathered}
$$


thus closing the system of equations for the scattering correction.

We solve (8) for the Green function on the left-hand side, substitute the result into (6), and rearrange and sum over $\beta$ to obtain, again for $i \neq k$,

$$
\left\langle\left\langle\delta n_{i \downarrow}^{\alpha} c_{k \uparrow} ; c_{j \uparrow}^{\dagger}\right\rangle\right\rangle_{\epsilon}=g_{\uparrow}(\epsilon) \sum_{l} t_{k l}\left\langle\left\langle\delta n_{i \downarrow}^{\alpha} c_{l \uparrow} ; c_{j \uparrow}^{\dagger}\right\rangle\right\rangle_{\epsilon},
$$

where $g_{\uparrow}(\epsilon)$ is the atomic-limit Green function presented in (幽). In the appendix of [10] Hubbard solved this equation in terms of $\left\langle\left\langle\delta n_{i \downarrow}^{\alpha} c_{i \uparrow} ; c_{j \uparrow}^{\dagger}\right\rangle\right\rangle_{\epsilon}$ :

$$
\left\langle\left\langle\delta n_{i \downarrow}^{\alpha} c_{k \uparrow} ; c_{j \uparrow}^{\dagger}\right\rangle\right\rangle_{\epsilon}=\left(\sum_{l} W_{k l}^{i \uparrow}(\epsilon) t_{l i}\right)\left\langle\left\langle\delta n_{i \downarrow}^{\alpha} c_{i \uparrow} ; c_{j \uparrow}^{\dagger}\right\rangle\right\rangle_{\epsilon}, \quad(i \neq k)
$$

where $W_{k l}^{i \sigma}$ is defined by

$$
\begin{aligned}
& W_{k l}^{i \sigma}(\epsilon)=\tilde{g}_{\sigma}^{k l}(\epsilon)-\frac{\tilde{g}_{\sigma}^{k i}(\epsilon) \tilde{g}_{\sigma}^{i l}(\epsilon)}{\tilde{g}_{\sigma}^{i i}(\epsilon)} \\
& \tilde{g}_{\sigma}^{i j}(\epsilon)=\frac{1}{N} \sum_{\vec{k}} \frac{\exp \left(\mathrm{i} \vec{k} \cdot \vec{R}_{i j}\right)}{g_{\sigma}(\epsilon)^{-1}-t_{\vec{k}}} .
\end{aligned}
$$

It is easy to check Hubbard's solution: from (11b) it may be shown that $g_{\sigma} \sum_{k} t_{i k} \tilde{g}_{\sigma}^{k j}=$ $\tilde{g}_{\sigma}^{i j}-g_{\sigma} \delta_{i j}$ and hence that $g_{\sigma} \sum_{l} t_{k l} W_{l j}^{i \sigma}=W_{k j}^{i \sigma}-g_{\sigma} \delta_{k j}$ for $i \neq k$, and this can be used to verify that substituting (10) into (9) does indeed give a solution.

As in Hubbard's case $\tilde{g}_{\sigma}^{i j}$ is a zeroth order approximation to $G_{\sigma}^{i j}$ in which both scattering and resonance broadening corrections are neglected, and following Hubbard we make the self-consistent replacement $\tilde{g}_{\uparrow}^{i j} \mapsto G_{\uparrow}^{i j}$; this is the essential self-consistency step of the CPA. From (10) the scattering correction term in (5) is given by

$$
\sum_{k} t_{i k}\left\langle\left\langle\delta n_{i \downarrow}^{\alpha} c_{k \uparrow} ; c_{j \uparrow}^{\dagger}\right\rangle\right\rangle_{\epsilon}=J_{\uparrow}(\epsilon)\left\langle\left\langle\delta n_{i \downarrow}^{\alpha} c_{i \uparrow} ; c_{j \uparrow}^{\dagger}\right\rangle\right\rangle_{\epsilon}
$$

where $J_{\sigma}(\epsilon)=\sum_{k l} t_{i k} W_{k l}^{i \sigma}(\epsilon) t_{l i}$. A result that will be useful later is

$$
J_{\sigma}(\epsilon)=\epsilon-\Sigma_{\sigma}(\epsilon)-G_{\sigma}(\epsilon)^{-1}
$$

which holds if the self-energy $\Sigma_{\sigma}(\epsilon)$ is local - as is expected for a CPA. This result may easily be established by making Fourier transforms and using $G_{\sigma}^{i j}(\epsilon)=N^{-1} \sum_{\vec{k}} \exp (i \vec{k}$. $\left.\vec{R}_{i j}\right) G_{\vec{k} \sigma}(\epsilon)$. We now also define the useful quantity

$$
E_{\sigma}(\epsilon)=\epsilon-J_{\sigma}(\epsilon)
$$

It remains to find expressions for the unknown Green functions $S_{\uparrow}$ and $T_{\uparrow}$ in (5); since these represent propagation as $\uparrow$ - and $\downarrow$-spins respectively we neglect the commutators $\left[n_{i \downarrow}^{\alpha}, H_{0}\right]$ and $\left[n_{i \uparrow}^{\alpha}, H_{0}\right]$ in the respective equations of motion:

$$
\begin{gathered}
\epsilon S_{\uparrow}^{i j \alpha}(\epsilon) \approx\left\langle S_{i}^{z} n_{i \downarrow}^{\alpha}\right\rangle\left(\delta_{i j}+\sum_{k} t_{i k} G_{\uparrow}^{k j}(\epsilon)\right)+\sum_{k} t_{i k}\left\langle\left\langle\delta\left(n_{i \downarrow}^{\alpha} S_{i}^{z}\right) c_{k \uparrow} ; c_{j \uparrow}^{\dagger}\right\rangle\right\rangle_{\epsilon}-\frac{J}{2} \delta_{\alpha+} T_{\uparrow}^{i j \alpha}(\epsilon) \\
-\frac{J}{2}\left\langle\left\langle n_{i}^{\alpha}\left(\left(S_{i}^{z}\right)^{2} c_{i \uparrow}+S_{i}^{z} S_{i}^{-} c_{i \downarrow}\right) ; c_{j \uparrow}^{\dagger}\right\rangle\right\rangle_{\epsilon} \\
\epsilon T_{\uparrow}^{i j \alpha}(\epsilon) \approx-\alpha\left\langle S_{i}^{-} \sigma_{i}^{+}\right\rangle\left(\delta_{i j}+\sum_{k} t_{i k} G_{\uparrow}^{k j}(\epsilon)\right)
\end{gathered}
$$




$$
\begin{aligned}
& +\sum_{k} t_{i k}\left\langle\left\langle\left(n_{i \uparrow}^{\alpha} S_{i}^{-} c_{k \downarrow}+\alpha\left\langle S_{i}^{-} \sigma_{i}^{+}\right\rangle c_{k \uparrow}\right) ; c_{j \uparrow}^{\dagger}\right\rangle\right\rangle_{\epsilon} \\
& -\frac{J}{2}\left(\alpha S_{\uparrow}^{i j \alpha}(\epsilon)-\delta_{\alpha-} T_{\uparrow}^{i j \alpha}(\epsilon)+S(S+1) G_{\uparrow}^{i j \alpha}(\epsilon)\right) \\
& +\frac{J}{2}\left\langle\left\langle n_{i}^{\alpha}\left(\left(S_{i}^{z}\right)^{2} c_{i \uparrow}+S_{i}^{z} S_{i}^{-} c_{i \downarrow}\right) ; c_{j \uparrow}^{\dagger}\right\rangle\right\rangle_{\epsilon} \cdot
\end{aligned}
$$

The second terms on the right-hand sides of these equations are the scattering corrections; the scattering correction in (15) is of the same form as in (5), but in (16) it is more complicated. It is not clear what the average 'zeroth order' Green function should be here, but we have chosen it to be $-\alpha\left\langle S_{i}^{-} \sigma_{i}^{+}\right\rangle G_{\uparrow}^{k j}(\epsilon)$ as this makes the first terms on the right-hand sides of (5), (15), and (16) all of the same form; it turns out that this is necessary for the consistency of the approximation. The last Green function in (15) and (16) involves higher order spin operators and is in general unknown; this term must be treated using an approximation which is exact in the atomic limit.

The scattering correction of (15) may be treated in the same way as that of (5); the only difference is that $\delta n_{i \downarrow}^{\alpha}$ is replaced by $\delta\left(n_{i \downarrow}^{\alpha} S_{i}^{z}\right)$, hence

$$
\sum_{k} t_{i k}\left\langle\left\langle\delta\left(n_{i \downarrow}^{\alpha} S_{i}^{z}\right) c_{k \uparrow} ; c_{j \uparrow}^{\dagger}\right\rangle\right\rangle_{\epsilon} \approx J_{\uparrow}(\epsilon)\left\langle\left\langle\delta\left(n_{i \downarrow}^{\alpha} S_{i}^{z}\right) c_{i \uparrow} ; c_{j \uparrow}^{\dagger}\right\rangle\right\rangle_{\epsilon} .
$$

It is less obvious how to treat the scattering correction of (16). Instead of giving a rigorous derivation we proceed by analogy and make the apparently reasonable approximation

$$
\begin{aligned}
\sum_{k} t_{i k}\left\langle\left\langle\left(n_{i \uparrow}^{\alpha} S_{i}^{-} c_{k \downarrow}+\alpha\left\langle S_{i}^{-} \sigma_{i}^{+}\right\rangle c_{k \uparrow}\right) ; c_{j \uparrow}^{\dagger}\right\rangle\right\rangle_{\epsilon} \\
\quad \approx J_{\downarrow}(\epsilon)\left\langle\left\langle n_{i \uparrow}^{\alpha} S_{i}^{-} c_{i \downarrow} ; c_{j \uparrow}^{\dagger}\right\rangle\right\rangle_{\epsilon}+J_{\uparrow}(\epsilon)\left\langle\left\langle\alpha\left\langle S_{i}^{-} \sigma_{i}^{+}\right\rangle c_{i \uparrow} ; c_{j \uparrow}^{\dagger}\right\rangle\right\rangle_{\epsilon} \\
=J_{\downarrow}(\epsilon) T_{\uparrow}^{i j \alpha}(\epsilon)+\alpha\left\langle S_{i}^{-} \sigma_{i}^{+}\right\rangle J_{\uparrow}(\epsilon) G_{\uparrow}^{i j}(\epsilon) .
\end{aligned}
$$

It will be seen later that this is consistent with the rest of the derivation, leading for instance to a local self-energy as expected in a CPA.

The system of equations of motion is now closed apart from the Green function $\left\langle\left\langle n_{i}^{\alpha}\left(\left(S_{i}^{z}\right)^{2} c_{i \uparrow}+S_{i}^{z} S_{i}^{-} c_{i \downarrow}\right) ; c_{j \uparrow}^{\dagger}\right\rangle\right\rangle_{\epsilon}$ appearing in (15) and (16). In general we will have to write more equations of motion to close the system. This is straightforward to do if we make approximations analogous to those used to obtain the equations of motion for $S_{\uparrow}$ and $T_{\uparrow}$. Then the Green functions $\left\langle\left\langle n_{i}^{\alpha}\left(S_{i}^{z}\right)^{m} c_{i \uparrow} ; c_{j \uparrow}^{\dagger}\right\rangle\right\rangle_{\epsilon}$ and $\left\langle\left\langle n_{i}^{\alpha}\left(S_{i}^{z}\right)^{m-1} S_{i}^{-} c_{i \downarrow} ; c_{j \uparrow}^{\dagger}\right\rangle\right\rangle_{\epsilon}$ and the expectations $\left\langle\left(S_{i}^{z}\right)^{m} n_{i \downarrow}^{-}\right\rangle$and $\left\langle\left(S_{i}^{z}\right)^{m-1} S_{i}^{-} \sigma_{i}^{+}\right\rangle$for $m=1, \ldots, 2 S$ are brought into the system of equations. The resulting system of $4 S+1$ equations per spin is complicated to solve self-consistently for large $S$ however, so we will restrict ourselves to simple special cases in which we do not need any more equations of motion; in the next three subsections we will consider the paramagnetic state in zero magnetic field for arbitrary $S$, the case $S=1 / 2$ for arbitrary magnetization, and the case of saturated ferromagnetism for arbitrary $S$.

Our approximation scheme is now complete and is entirely self-consistent, i.e. all the expectations appearing in the system of equations can be obtained from the Green 
functions in the system via the relation [13]

$$
\langle B A\rangle=-\int_{-\infty}^{\infty} \frac{\mathrm{d} \epsilon}{\pi} f(\epsilon-\mu) \operatorname{Im}\langle\langle A ; B\rangle\rangle_{\epsilon}
$$

where $f(\epsilon)=1 /(1+\exp (\beta \epsilon))$ is the Fermi function and $\mu$ is the chemical potential. Also, since all the approximations made above have been in terms proportional to $t_{i j}$ the approximation is exact in the atomic limit for all $S$ and $n$ as required. The system is assumed to be homogeneous, so we now drop the site indices of the expectations.

\subsection{Paramagnetism}

In the case of paramagnetism with zero field $J_{\uparrow}=J_{\downarrow}$. Hence after substituting (17) and (18b) in (15) and (16), respectively, we can add the resulting equations so that $S^{i j \alpha}$ and $T^{i j \alpha}$ occur only in the combination $S^{i j \alpha}+T^{i j \alpha}$ :

$$
\begin{gathered}
\epsilon\left(S^{i j \alpha}(\epsilon)+T^{i j \alpha}(\epsilon)\right)=\left\langle S^{z} n_{\downarrow}^{\alpha}-\alpha S^{-} \sigma^{+}\right\rangle\left(\delta_{i j}+\sum_{k} t_{i k} G^{k j}(\epsilon)-J(\epsilon) G^{i j}(\epsilon)\right) \\
+\left(J(\epsilon)-\frac{J \alpha}{2}\right)\left(S^{i j \alpha}(\epsilon)+T^{i j \alpha}(\epsilon)\right)-\frac{J S(S+1)}{2} G^{i j \alpha}(\epsilon) .
\end{gathered}
$$

Since $S^{i j \alpha}$ and $T^{i j \alpha}$ also enter (5) as $S^{i j \alpha}+T^{i j \alpha}$ we have now closed the system. We substitute (12) and (20) into (5) and rearrange to obtain

$$
G^{i j}(\epsilon)=\left(\delta_{i j}+\sum_{k} t_{i k} G^{k j}(\epsilon)-J(\epsilon) G^{i j}(\epsilon)\right) \tilde{G}(\epsilon)
$$

where $\tilde{G}$ is defined by

$$
\tilde{G}(\epsilon)=\sum_{\alpha= \pm} \frac{(E(\epsilon)+\alpha J / 2)(n / 2)^{\alpha}+J \alpha / 2\langle\vec{S} \cdot \vec{\sigma}\rangle}{(E(\epsilon)-\alpha J S / 2)(E(\epsilon)+\alpha J(S+1) / 2)},
$$

$E(\epsilon)=\epsilon-J(\epsilon)$, and $(n / 2)^{\alpha}=\delta_{\alpha-}+\alpha n / 2$. The spin symmetry of the paramagnetic state has been used to simplify the expectations appearing in (22).

Taking Fourier transforms and solving for $G_{\vec{k}}(21)$ becomes

$$
G_{\vec{k}}(\epsilon)=\frac{1}{J(\epsilon)+\tilde{G}(\epsilon)^{-1}-t_{\vec{k}}} .
$$

Since in general $G_{\vec{k}}(\epsilon)=\left(\epsilon-t_{\vec{k}}-\Sigma_{\vec{k}}(\epsilon)\right)^{-1}$ this implies that the self-energy is local, $\Sigma_{\vec{k}}(\epsilon)=\Sigma(\epsilon)$, and so using expression (13) for $J(\epsilon)$ (23) becomes

$$
G_{\vec{k}}(\epsilon)=\frac{1}{\epsilon-t_{\vec{k}}-\Sigma(\epsilon)+\tilde{G}(\epsilon)^{-1}-G(\epsilon)^{-1}},
$$

and for consistency in this equation we must have $G=\tilde{G}$. The existence of this simple self-consistency condition defining $G$ is our main justification for the approximations made for the scattering correction of (16). If the approximations are changed we will not obtain a consistent expression defining a local self-energy.

In the low density limit $n \rightarrow 0$ (22) reduces to

$$
G(\epsilon)=\frac{E(\epsilon)-J / 2}{(E(\epsilon)+J S / 2)(E(\epsilon)-J(S+1) / 2)},
$$

which is Kubo's [14] equation for the paramagnetic state Green function as required. 


\subsection{The $S=1 / 2$ case for arbitrary magnetization}

For $S=1 / 2$ we have $\left(S_{i}^{z}\right)^{2}=1 / 4$ and $S_{i}^{z} S_{i}^{-}=-1 / 2 S_{i}^{-}$for arbitrary magnetization, so the last Green function in (15) and (16) may be simplified:

$$
\left\langle\left\langle n_{i}^{\alpha}\left(\left(S_{i}^{z}\right)^{2} c_{i \uparrow}+S_{i}^{z} S_{i}^{-} c_{i \downarrow}\right) ; c_{j \uparrow}^{\dagger}\right\rangle\right\rangle_{\epsilon}=\frac{1}{4} G_{\uparrow}^{i j \alpha}(\epsilon)-\frac{1}{2} T_{\uparrow}^{i j \alpha}(\epsilon),
$$

closing the system. Substituting the scattering corrections (12), (17), and (18b) into the equations of motion (5), (15), and (16) the system of equations reduces to

$$
\begin{aligned}
& E_{\uparrow} G_{\uparrow}^{i j \alpha}=\left\langle n_{\downarrow}^{\alpha}\right\rangle \lambda_{\uparrow}^{i j}-\frac{J}{2}\left(S_{\uparrow}^{i j \alpha}+T_{\uparrow}^{i j \alpha}\right) \\
& E_{\uparrow} S_{\uparrow}^{i j \alpha}=\left\langle S^{z} n_{\downarrow}^{\alpha}\right\rangle \lambda_{\uparrow}^{i j}-\frac{J}{8} G_{\uparrow}^{i j \alpha}-\frac{J \alpha}{4} T_{\uparrow}^{i j \alpha} \\
& E_{\downarrow}^{\alpha} T_{\uparrow}^{i j \alpha}=-\alpha\left\langle S^{-} \sigma^{+}\right\rangle \lambda_{\uparrow}^{i j}-\frac{J}{4} G_{\uparrow}^{i j \alpha}-\frac{J \alpha}{2} S_{\uparrow}^{i j \alpha},
\end{aligned}
$$

where

$$
\lambda_{\sigma}^{i j}(\epsilon)=\delta_{i j}+\sum_{k} t_{i k} G_{\sigma}^{k j}(\epsilon)-J_{\sigma}(\epsilon) G_{\sigma}^{i j}(\epsilon),
$$

$E_{\sigma}(\epsilon)=\epsilon-J_{\sigma}(\epsilon)$, and $E_{\sigma}^{\alpha}=E_{\sigma}+J \alpha / 4$. These equations can be solved using a similar method to that of the previous subsection, with an analogous self-consistency condition occurring, and the local Green function is given by

$G_{\uparrow}(\epsilon)=\sum_{\alpha= \pm} \frac{\left\langle n_{\downarrow}^{\alpha}\right\rangle\left(E_{\uparrow}(\epsilon) E_{\downarrow}^{\alpha}(\epsilon)-J^{2} / 8\right)-J / 2\left(\left\langle S^{z} n_{\downarrow}^{\alpha}\right\rangle E_{\downarrow}^{-\alpha}(\epsilon)-\alpha\left\langle S^{-} \sigma^{+}\right\rangle E_{\uparrow}^{-\alpha}(\epsilon)\right)}{E_{\uparrow}^{-\alpha}(\epsilon)\left(E_{\uparrow}^{\alpha}(\epsilon) E_{\downarrow}^{\alpha}(\epsilon)-J^{2} / 4\right)}$.

The expectations $\left\langle n_{\downarrow}^{\alpha}\right\rangle,\left\langle S^{z} n_{\downarrow}^{\alpha}\right\rangle$, and $\left\langle S^{-} \sigma^{+}\right\rangle$may be calculated self-consistently using (27d) $-(27 \mathrm{~d})$ and (19).

If we set $n=0$ (29) reduces to

$$
G_{\uparrow}(\epsilon)=\frac{E_{\uparrow}(\epsilon) E_{\downarrow}^{-}(\epsilon)-J^{2} / 8-J / 2\left\langle S^{z}\right\rangle E_{\downarrow}^{+}(\epsilon)}{E_{\uparrow}^{+}(\epsilon)\left[E_{\uparrow}^{-}(\epsilon) E_{\downarrow}^{-}(\epsilon)-J^{2} / 4\right]} .
$$

For $S=1 / 2$ the probabilities of a local spin being up or down are given by $P\left(S^{z}=\right.$ $\pm 1 / 2)=1 / 2 \pm\left\langle S^{z}\right\rangle$, and this fact may be used to show that (30) is equal to Kubo's equation 114 for $G_{\uparrow}$ in the $S=1 / 2$ case. Agreement with Kubo's approximation in the case of arbitrary magnetization is a much more stringent condition on our CPA than agreement just in the paramagnetic case, so this gives us confidence that our approximation is indeed a many-body extension of Kubo's.

\subsection{Saturated ferromagnetism for arbitrary $S$}

If we assume the existence of a saturated ferromagnetic state where all local spins and conduction electron spins are aligned parallel to the positive $z$-axis substantial simplification occurs. The expectations occurring in the $\uparrow$ - and $\downarrow$-spin systems are given by

$$
\begin{array}{lll}
\left\langle n_{\downarrow}^{\alpha}\right\rangle=\delta_{\alpha-} & \left\langle S^{z} n_{\downarrow}^{\alpha}\right\rangle=S \delta_{\alpha-} & \left\langle S^{-} \sigma^{+}\right\rangle=0 \\
\left\langle n_{\uparrow}^{\alpha}\right\rangle=\delta_{\alpha-}+\alpha n & \left\langle-S^{z} n_{\uparrow}^{\alpha}\right\rangle=-S\left(\delta_{\alpha-}+\alpha n\right) & \left\langle S^{+} \sigma^{-}\right\rangle=0,
\end{array}
$$


and the Green functions $G_{\sigma}, S_{\sigma}$, and $T_{\sigma}$ may be simplified using the relations

$$
\begin{array}{ll}
G_{\uparrow}^{i j+}=T_{\uparrow}^{i j \alpha}=0 & S_{\uparrow}^{i j \alpha}=S \delta_{\alpha-} G_{\uparrow}^{i j} \\
T_{\downarrow}^{i j+}=0 & S_{\downarrow}^{i j \alpha}=-S G_{\downarrow}^{i j \alpha} .
\end{array}
$$

The higher order Green functions are given by

$$
\begin{aligned}
& \left\langle\left\langle n_{i}^{\alpha}\left(\left(S_{i}^{z}\right)^{2} c_{i \uparrow}+S_{i}^{z} S_{i}^{-} c_{i \downarrow}\right) ; c_{j \uparrow}^{\dagger}\right\rangle\right\rangle_{\epsilon}=S^{2} \delta_{\alpha-} G_{\uparrow}^{i j} \\
& \left\langle\left\langle n_{i}^{\alpha}\left(\left(S_{i}^{z}\right)^{2} c_{i \downarrow}-S_{i}^{z} S_{i}^{+} c_{i \uparrow}\right) ; c_{j \downarrow}^{\dagger}\right\rangle\right\rangle_{\epsilon}=S^{2} G_{\downarrow}^{i j \alpha}-S \delta_{\alpha-} T_{\downarrow}^{i j} .
\end{aligned}
$$

The system therefore closes without needing any more equations. After simplification the set of equations of motion (for both spin types) reduces to

$$
\begin{aligned}
& \left(E_{\uparrow}+\frac{J S}{2}\right) G_{\uparrow}^{i j}=\lambda_{\uparrow}^{i j} \\
& \left(E_{\downarrow}-\frac{J S}{2}\right) G_{\downarrow}^{i j+}=n \lambda_{\downarrow}^{i j} \\
& \left(E_{\downarrow}-\frac{J S}{2}\right) G_{\downarrow}^{i j-}=(1-n) \lambda_{\downarrow}^{i j}-\frac{J}{2} T_{\downarrow}^{i j} \\
& \left(E_{\uparrow}+\frac{J(S-1)}{2}\right) T_{\downarrow}^{i j}=-J S G_{\downarrow}^{i j-},
\end{aligned}
$$

with the other equations satisfied automatically. Here $\lambda_{\sigma}^{i j}$ and $E_{\sigma}$ are defined as in the previous subsection.

We can solve these equations in the same way as in the previous two subsections, with the usual self-consistency condition similar to (24) applying, and the local Green functions are given by

$$
\begin{aligned}
G_{\uparrow} & =\frac{1}{E_{\uparrow}+J S / 2} \\
G_{\downarrow} & =\frac{n}{E_{\downarrow}-J S / 2}+\frac{1-n}{E_{\downarrow}-J S / 2-J^{2} S / 2\left(E_{\uparrow}+J(S-1) / 2\right)^{-1}} .
\end{aligned}
$$

The $\uparrow$-spin Green function here is just the free Green function shifted in energy, as might be expected. In the $n \rightarrow 0$ limit these equations reduce to those of Kubo [14, providing a further check on our approximation. It is shown in the next section that these Green functions are in fact not consistent with the initial assumption of a saturated ferromagnetic state.

\section{CPA spectral function}

In this section we study the DOS, first for the saturated ferromagnetic state and then for the paramagnetic state. We make the approximation of replacing the true DOS $D_{\text {c }}$ for a simple cubic lattice with the elliptic DOS, $D_{\mathrm{e}}(\epsilon)=2 /\left(\pi W^{2}\right) \sqrt{W^{2}-\epsilon^{2}}$ where $2 W$ is the bandwidth. For an elliptic DOS the free Green function is given by $G_{0}(\epsilon)=2 / W^{2}\left(\epsilon-\sqrt{\epsilon^{2}-W^{2}}\right)$. Since the self-energy $\Sigma_{\sigma}(\epsilon)$ is local the full Green function is given by $G_{\sigma}(\epsilon)=G_{0}\left(\epsilon-\Sigma_{\sigma}(\epsilon)\right)$, and it follows immediately that $J_{\sigma}=W^{2} / 4 G_{\sigma}$. The Green function equations therefore become algebraic in this approximation, greatly 
simplifying the calculations. The elliptic DOS is also a fairly good approximation to the true DOS, unlike the Lorentzian DOS $D_{\mathrm{l}}(\epsilon)=(W / \pi) /\left(W^{2}+\epsilon^{2}\right)$ considered by Furukawa [3]. In the infinite dimensional limit of a hypercubic lattice with nearest neighbour hopping - the scenario considered by Furukawa - it may be shown that the true DOS is a Gaussian if the hopping $t_{i j}$ is scaled as the inverse square root of the number of dimensions [15]. With the scaling appropriate to three dimensions the Gaussian DOS is given by $D_{\mathrm{g}}(\epsilon)=(3 / \pi)^{1 / 2} \exp \left[-3(\epsilon / W)^{2}\right] / W$. These DOS's are compared in figure 1 .

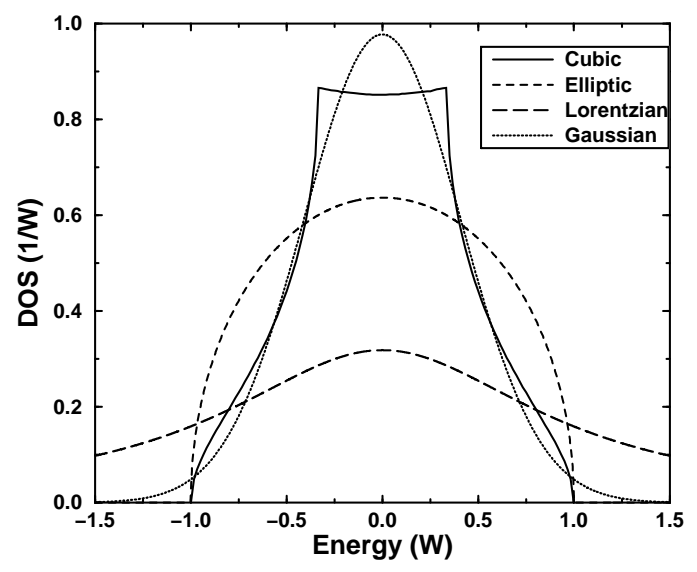

Figure 1. The DOS's $D_{\mathrm{c}}, D_{\mathrm{e}}, D_{\mathrm{l}}$, and $D_{\mathrm{g}}$ plotted in units of $W$.

\subsection{Saturated ferromagnetism}

We study the ferromagnetic state with complete spin alignment in the strong-coupling $J \rightarrow \infty$ limit which is most favourable to ferromagnetism, shifting the energy origin by $-J S / 2$ in order to have the zero of energy in the lowest band. Equations (35a) and (35b) become

$$
\begin{aligned}
G_{\uparrow} & =1 / E_{\uparrow} \\
G_{\downarrow} & =\frac{1-n}{E_{\downarrow}+2 S E_{\uparrow}} .
\end{aligned}
$$

The DOS of this state is plotted in figure 2 for $S=1 / 2$ and various $n$. Since the $\uparrow$ and $\downarrow$-spin DOS's are nonzero for the same range of energies it is clear that consistent saturated ferromagnetism does not occur within our approximation for any $n$. It may easily be shown that this is true for any finite $S$. Since strong ferromagnetism is expected to occur in the DE model for at least some parameter values this is a limitation of our approximation and suggests that our CPA may not be very good at low temperatures. Of course in common with the usual alloy CPA we cannot obtain a true Fermi liquid groundstate in our CPA - the imaginary part of the self-energy does not vanish at the Fermi surface at $T=0$ - so we only expect our CPA to describe the DE model well 
at finite (but not necessarily large) temperatures where this is not a problem. The possibility of weak ferromagnetism occurring in our approximation will be discussed elsewhere; preliminary work on the magnetic susceptibility has already appeared in [7]-[9].

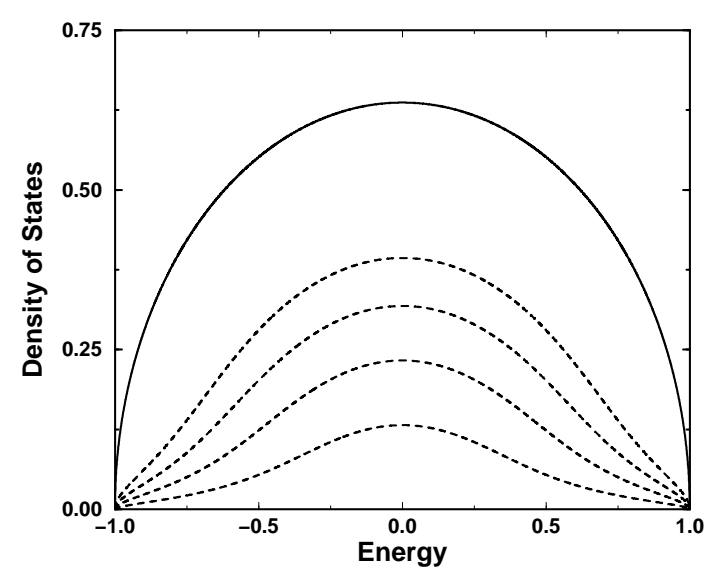

Figure 2. The $\uparrow$-spin (solid curve, independent of $n$ ) and $\downarrow$-spin (dashed curves, $n=0,0.25,0.5,0.75$, DOS decreasing with increasing $n$ ) DOS's in the saturated ferromagnetic state for $S=1 / 2$ and $J=\infty$. Energy units of $W$ are used.

Note that the weights of the $\uparrow$ - and $\downarrow$-spin bands here are 1 and $(1-n) /(2 S+1)$ respectively, so in the classical spin limit $S \rightarrow \infty$ there is no possibility of $\downarrow$-spin weight occurring near the Fermi level. In fact for $S \rightarrow \infty$ the saturated state is always stable against spin reversal but for finite $S$ this is not always the case [16].

\subsection{Paramagnetism}

We now consider the zero field paramagnetic state. It may be shown that $\langle\vec{S} \cdot \vec{\sigma}\rangle \rightarrow n S / 2$ as $J \rightarrow \infty$, and $\langle\vec{S} \cdot \vec{\sigma}\rangle$ will be very near to this limit as long as $J S \gtrsim 2 W$. We make this approximation in figure 3, in which the paramagnetic state DOS is plotted for $S=3 / 2$ and $J=4 W$ for various $n$; this has the effect of removing the weak band centred on $-J(S+1) / 2$. It may be seen that as $n$ increases from 0 the band near $J(S+1) / 2$ is reduced in weight and a new band appears near $J S / 2$, until at $n=1$ no weight remains in the band near $J(S+1) / 2$. The weight in the band near $-J S / 2$ is $(S+1-n / 2) /(2 S+1)$ per spin, so if $J S$ is sufficiently large to separate the bands $(J S \gtrsim 2 W)$ this band will just be filled at $n=1$ producing a Mott insulator, as discussed in section 2 .

We can understand figure 3 by expanding (22) in partial fractions,

$G(\epsilon)=\frac{1}{2 S+1} \sum_{\alpha= \pm}\left(\frac{(S+1)(n / 2)^{\alpha}+\langle\vec{S} \cdot \vec{\sigma}\rangle}{E(\epsilon)-\alpha J S / 2}+\frac{S(n / 2)^{\alpha}-\langle\vec{S} \cdot \vec{\sigma}\rangle}{E(\epsilon)+\alpha J(S+1) / 2}\right)$.

Comparing this equation with (屯) in the paramagnetic state we see that $G(\epsilon)=g(E(\epsilon))$, 

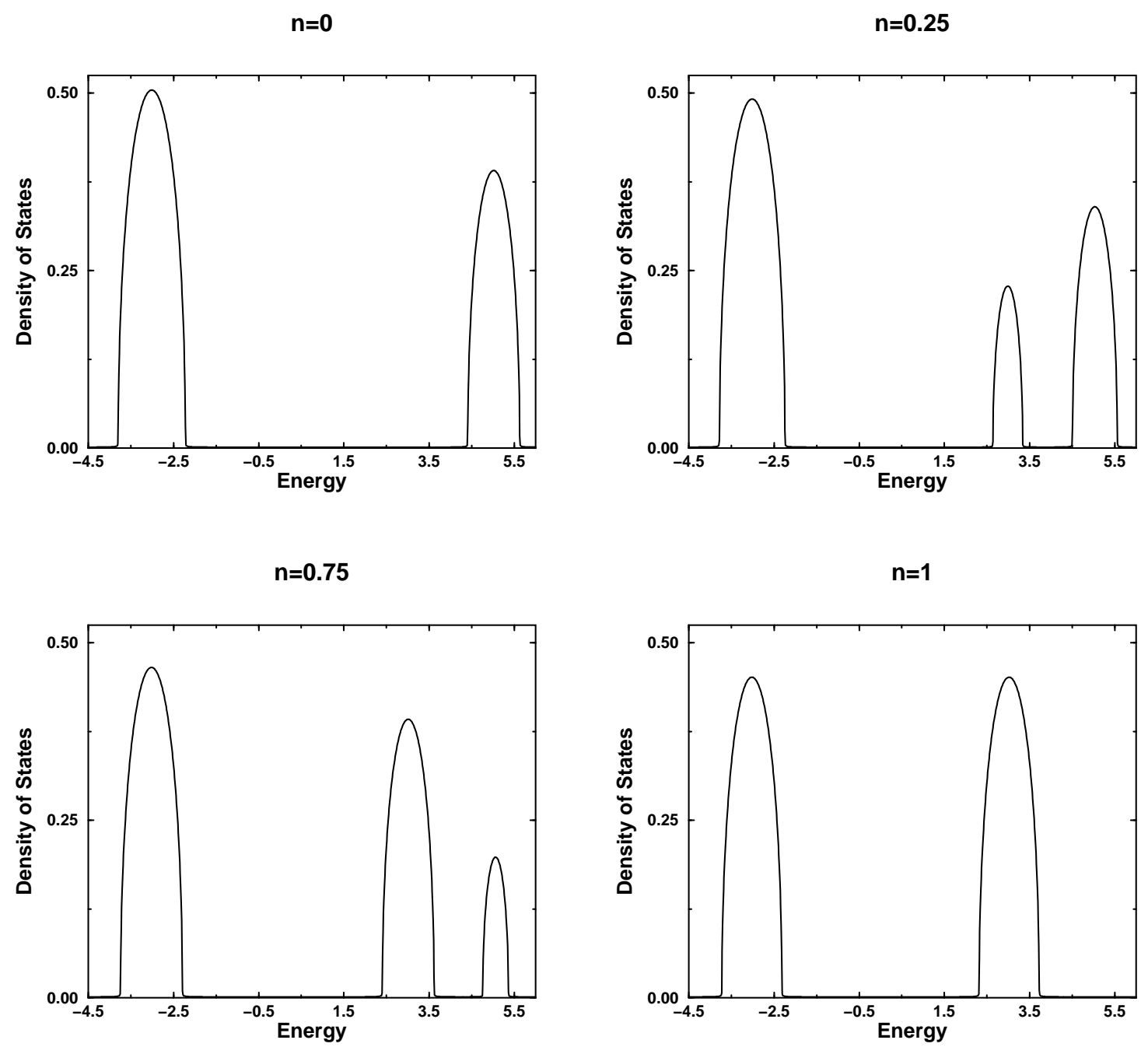

Figure 3. The DOS in the paramagnetic state for $S=3 / 2, J=4 W$, and $n=0,0.25,0.75$ and 1. Energy units of $W$ are used.

and since $E(\epsilon) \rightarrow \epsilon$ as $t_{i j} \rightarrow 0$ it is clear how the bands centred on the peaks of the atomic limit spectral function arise as $t_{i j}$ is switched on.

In the strong-coupling limit $J \rightarrow \infty$, which is taken with the energy origin shifted by $-J S / 2$, (37) simplifies to

$$
G(\epsilon)=\frac{(S+1-n / 2) /(2 S+1)}{E(\epsilon)},
$$

which corresponds to an elliptical band of weight $(S+1-n / 2) /(2 S+1)$ and bandwidth $2 W \sqrt{(S+1-n / 2) /(2 S+1)}$. The band-narrowing factor is expected to depend upon the form of the bare DOS, as pointed out by Kubo [12], but it is interesting to note that ours is the square root of the factor obtained by Brunton and Edwards [16] using a different method. 


\section{Comparison with the hole CPA}

In [17 Kubo and Ohata used a canonical transformation to derive an effective Hamiltonian $H^{\prime}$ for the $J \rightarrow \infty$ limit of the DE model. They then mapped $H^{\prime}$ onto an effective Hamiltonian $H^{\prime \prime}$ for holes, in which conduction holes hop with spins aligned antiparallel to local spins $(S+1 / 2)$. In [12 Kubo studied $H^{\prime \prime}$ for $n=1$, using a dynamical CPA similar to his $n=0$ CPA mentioned above. There is no obvious reason for one of these CPA's to be a better approximation than the other, so we would like our many-body CPA to agree with both of Kubo's approximations in the appropriate limits. In this section we set $n=1$ and $J=\infty$ in our $S=1 / 2$ Green function equation (29) and compare the result with Kubo's hole CPA.

For $S=1 / 2$ Kubo's $n=1$ Green function is given by

$$
G_{\uparrow}=\frac{3 / 2\left(\tilde{E}_{\downarrow}+\tilde{E}_{\uparrow}\right)+\left(2 \tilde{E}_{\downarrow}+\tilde{E}_{\uparrow}\right)\left\langle S^{z}+\sigma^{z}\right\rangle+2\left(\tilde{E}_{\downarrow}-\tilde{E}_{\uparrow}\right)\left\langle S^{z} \sigma^{z}\right\rangle}{2 / 3\left(2 \tilde{E}_{\downarrow}+\tilde{E}_{\uparrow}\right)\left(2 \tilde{E}_{\uparrow}+\tilde{E}_{\downarrow}\right)}
$$

where $\tilde{E}_{\sigma}(\epsilon)=\epsilon-3 W^{2} / 8 G_{\sigma}(\epsilon)$. If we set $n=1$ and $J=\infty$ in (29) we obtain

$$
G_{\uparrow}=\frac{\left(E_{\downarrow}+3 E_{\uparrow}\right) / 4+\left(E_{\downarrow}+E_{\uparrow}\right)\left\langle S^{z}+\sigma^{z}\right\rangle / 2+\left(E_{\downarrow}-E_{\uparrow}\right)\left\langle S^{z} \sigma^{z}\right\rangle}{E_{\uparrow}\left(E_{\uparrow}+E_{\downarrow}\right)} .
$$

For $n=1$ and $J=\infty$ the relation $\left\langle S^{z}\right\rangle=\left\langle\sigma^{z}\right\rangle$ holds, and we have used this to write both expectations as $\left\langle S^{z}+\sigma^{z}\right\rangle / 2$. Unfortunately the Green functions defined by (39) and (40) are not equal; this may be seen by considering the zero-field paramagnetic state where they reduce to

$$
\begin{aligned}
& G(\epsilon)=\frac{1 / 2}{\tilde{E}(\epsilon)}=\frac{1 / 2}{\epsilon-3 W^{2} / 8 G(\epsilon)} \\
& G(\epsilon)=\frac{1 / 2}{E(\epsilon)}=\frac{1 / 2}{\epsilon-W^{2} / 4 G(\epsilon)}
\end{aligned}
$$

respectively, and these equations correspond to bands with different widths. This appears to be a limitation of our CPA, which we would ideally like to interpolate continuously between Kubo's CPA's. A possible explanation for this disagreement is our neglect of resonance broadening corrections in section 3 .

We could attempt to include resonance broadening corrections in our approximation by writing more equations of motion for these terms. It is however difficult to find approximations that give a closed set of equations for the new Green functions introduced without spoiling the self-consistency of our approximation. An alternative is to use an interpolation scheme containing arbitrary parameters which are chosen to yield as many correct moments of the spectral function as possible [18].

We first note that an empirical modification of (14), of the form

$$
\begin{aligned}
& E_{\uparrow}(\epsilon)=\epsilon-\frac{W^{2}}{4}\left[G_{\uparrow}(\epsilon)+\frac{1}{2} G_{\downarrow}(\epsilon)\right] \\
& E_{\downarrow}(\epsilon)=\epsilon-\frac{3 W^{2}}{8} G_{\downarrow}(\epsilon),
\end{aligned}
$$

maps (40) for $G_{\uparrow}$ onto Kubo's equation (39). This modification is of the same type as Hubbard's resonance broadening correction to the strong coupling $(U \rightarrow \infty)$ limit of his 
model. This suggests that we should introduce an interpolation formula for $E_{\sigma}$ which reduces to (42a) and (42b) in the limits $n=0$ and $n=1$, respectively. Unfortunately so far work along these lines has not proved successful.

\section{Resistivity formula}

We now derive a formula for the DC resistivity $\rho$ of the paramagnetic state of our model, taking into account the cubic symmetry of the crystal and the local nature of our approximation. The Kubo formula [13] states that for a small electric field uniform in space but oscillatory in time with frequency $\omega, \vec{E}(\mathbf{r}, t)=\vec{E}_{0} \exp (-\mathrm{i} \omega t)$, the conductivity tensor $\sigma_{\mu \nu}(\omega)$ is given in terms of the current-current correlation function by

$$
\sigma_{\mu \nu}(\omega)=\frac{\mathrm{i} n e^{2}}{\Omega m \omega} \delta_{\mu \nu}+\frac{\mathrm{i} N \Omega}{\omega}\left\langle\left\langle J_{\mu} ; J_{\nu}\right\rangle\right\rangle_{\omega}
$$

where $\hbar=1, \Omega$ is the unit cell volume, $m$ and $-e$ are the electron mass and charge respectively, and the retarded Green function is used. $\vec{J}$ is the electric current density operator defined for a homogeneous system by

$$
\vec{J}=-\frac{e}{N \Omega} \sum_{\vec{k} \sigma} \vec{v}_{\vec{k}} n_{\vec{k} \sigma}
$$

where the velocity $\vec{v}_{\vec{k}}=\nabla_{\vec{k}} t_{\vec{k}}$. In our case the conductivity is a real scalar so from (43)

$$
\sigma=-N \Omega \lim _{\omega \rightarrow 0} \operatorname{Im}\left[\frac{\left\langle\left\langle J_{x} ; J_{x}\right\rangle\right\rangle_{\omega}}{\omega}\right] .
$$

Now from (44)

$$
\left\langle\left\langle J_{x} ; J_{x}\right\rangle\right\rangle_{\omega}=\frac{e^{2}}{3(N \Omega)^{2}} \sum_{\vec{k} \vec{k}^{\prime} \sigma \sigma^{\prime}} \vec{v}_{\vec{k}} \cdot \vec{v}_{\vec{k}^{\prime}}\left\langle\left\langle n_{\vec{k} \sigma} ; n_{\vec{k}^{\prime} \sigma^{\prime}}\right\rangle\right\rangle_{\omega},
$$

so we need an approximation to the two-electron Green function $\left\langle\left\langle n_{\vec{k} \sigma} ; n_{\vec{k}^{\prime} \sigma^{\prime}}\right\rangle\right\rangle_{\omega}$.

Since the self-energy is independent of momentum a reasonable approximation is to assume that the irreducible vertex function is also independent of momentum. In infinite dimensions where the self-energy is rigorously local the momentum-dependent contribution of the irreducible vertex function vanishes. In this case the contribution of the vertex correction to $\sigma$ vanishes owing to the different parities of $\vec{v}_{\vec{k}}$ and $t_{\vec{k}}$ in $\vec{k}$, and we can evaluate the two-electron Green function $\left\langle\left\langle n_{\vec{k} \sigma} ; n_{\vec{k} \sigma}\right\rangle\right\rangle_{\mathrm{i} \nu}$ in the bubble approximation, obtaining

$$
\begin{aligned}
\frac{1}{\beta} \sum_{m} G_{\vec{k} \sigma}\left(\mathrm{i} \omega_{m}+\mu\right) G_{\vec{k} \sigma}\left(\mathrm{i} \omega_{m}+\mathrm{i} \nu+\mu\right) \\
=\frac{1}{\beta} \int \mathrm{d} \epsilon \int \mathrm{d} \eta \sum_{m} \frac{A_{\vec{k} \sigma}(\epsilon) A_{\vec{k} \sigma}(\eta)}{\left(\mathrm{i} \omega_{m}+\mu-\epsilon\right)\left(\mathrm{i} \omega_{m}+\mathrm{i} \nu+\mu-\eta\right)}
\end{aligned}
$$

where the $\mathrm{i} \omega_{m}$ 's are odd Matsubara frequencies and $\mathrm{i} \nu$ is an even Matsubara frequency and the spectral representation of the one-electron Green function, $A_{\vec{k} \sigma}(\epsilon)=$ $-\operatorname{Im} G_{\vec{k} \sigma}(\epsilon) / \pi$, has been used. The sum over $m$ can be evaluated using Cauchy's residue theorem, and following analytic continuation to the real axis and a shift in $\eta$ we obtain

$$
\left\langle\left\langle n_{\vec{k} \sigma} ; n_{\vec{k} \sigma}\right\rangle\right\rangle_{\omega}=\int \mathrm{d} \epsilon \int \mathrm{d} \eta \frac{A_{\vec{k} \sigma}(\epsilon) A_{\vec{k} \sigma}(\eta+\epsilon)}{\omega-\eta}[f(\epsilon-\mu)-f(\epsilon+\eta-\mu)] .
$$


In the paramagnetic state $G$ is $T$-independent if we assume $\langle\vec{S} \cdot \vec{\sigma}\rangle=n S / 2$. The Fermi functions in (48) give the conductivity $\sigma$ a weak $T$-dependence but we neglect this dependence and calculate at $T=0$, considering our calculation to apply to the $T>T_{\mathrm{C}}$ state however. Equations (45), (46), and (48) then imply that

$$
\sigma \approx \frac{2 \pi e^{2}}{3 N \Omega} \sum_{\vec{k}} \vec{v}_{\vec{k}}^{2} A_{\vec{k}}(\mu)^{2} .
$$

Now for the simple cubic band $t_{\vec{k}}=-2 t\left[\cos \left(k_{x} a\right)+\cos \left(k_{y} a\right)+\cos \left(k_{z} a\right)\right]$, where $a$ is the lattice constant, and (49) can be simplified using Gauss' theorem:

$$
\sigma=\frac{2 \pi e^{2}}{3 N a} \sum_{\vec{k}} t_{\vec{k}} \phi\left(t_{\vec{k}}\right)
$$

where $\phi^{\prime}\left(t_{\vec{k}}\right)=A_{\vec{k}}(\mu)^{2}$. This is a legitimate definition since $A_{\vec{k}}$ depends on $\vec{k}$ only through $t_{\vec{k}}$ in the local approximation. We introduce the cubic bare DOS $D_{\mathrm{c}}(\epsilon)$, and (50) becomes

$$
\sigma=\frac{2 \pi e^{2}}{3 a} \int \mathrm{d} \epsilon \epsilon D_{\mathrm{c}}(\epsilon) \phi(\epsilon) .
$$

Integrating $A_{\epsilon}(\mu)^{2}$ with respect to $\epsilon$ it may be shown that

$\phi(\epsilon)=\frac{1}{2 \pi^{2}}\left[\frac{\epsilon+\Sigma^{\prime}(\mu)-\mu}{\left(\Sigma^{\prime \prime}(\mu)\right)^{2}+\left(\epsilon+\Sigma^{\prime}(\mu)-\mu\right)^{2}}+\frac{1}{\Sigma^{\prime \prime}(\mu)} \tan ^{-1}\left(\frac{\epsilon+\Sigma^{\prime}(\mu)-\mu}{\Sigma^{\prime \prime}(\mu)}\right)\right]$

where $\Sigma^{\prime}(\mu)$ and $\Sigma^{\prime \prime}(\mu)$ are the real and imaginary parts of $\Sigma(\mu)$ respectively.

If $D_{\mathrm{c}}(\epsilon)$ is replaced with the Gaussian $D_{\mathrm{g}}(\epsilon)$ corresponding to an infinite dimensional approximation [15], (51) may be simplified by integrating by parts:

$$
\sigma=\frac{\pi e^{2} W^{2}}{9 a} \int \mathrm{d} \epsilon D_{\mathrm{g}}(\epsilon) A_{\epsilon}(\mu)^{2}
$$

Note that this conductivity formula is of the same form as Furukawa's [3].

\section{Resistivity calculations}

In this section we will use formulas (51), (52), and (53) to calculate $\rho=\sigma^{-1}$, making simple analytic approximations for $D_{\mathrm{c}}(\epsilon)$. Note that in SI units (51) and (53) must be divided by $\hbar$.

\subsection{Elliptical DOS}

We first calculate $\rho$ for the elliptical DOS $D_{e}(\epsilon)$. We take the strong-coupling limit $J \rightarrow \infty$ for simplicity; the precise value of $J$ is unimportant in the strong-coupling regime $J S \gtrsim 2 W . \rho$ is plotted against $n$ for various $S$ in figure 4 . It may be seen that $\rho$ correctly diverges at $n=0$ and $n=1$, but the size of $\rho$ and $\partial \rho / \partial n$ for $n \sim 0.8$ are much too small to explain experiment, at least in some materials. For example Urushibara et al [19], who measured $\rho$ for $\mathrm{La}_{n} \mathrm{Sr}_{1-n} \mathrm{MnO}_{3}$, found (for $T$ well above $T_{\mathrm{C}}$ ) that $\rho \sim 20 \mathrm{~m} \Omega \mathrm{cm}$ for $n \sim 0.8$ and that $\rho$ drops by about an order of magnitude as $n$ 
is reduced from 0.85 to 0.7 . We therefore agree with Millis et al [U] that the bare DE model does not explain the paramagnetic state of the CMR materials. We also find that the $T$-dependence of $\rho$ is always of the metallic $\partial \rho / \partial T>0$ form in our approximation, whereas Urushibara et al find a crossover between metallic behaviour at $n=0.7$ to insulating behaviour at $n=0.85$. It should be noted that $\rho$ is independent of $W$ here, and the size of $J$ and $S$ and the choice of resistivity formula (51) or (53), using $D_{e}$ in both cases, does not have a large effect.

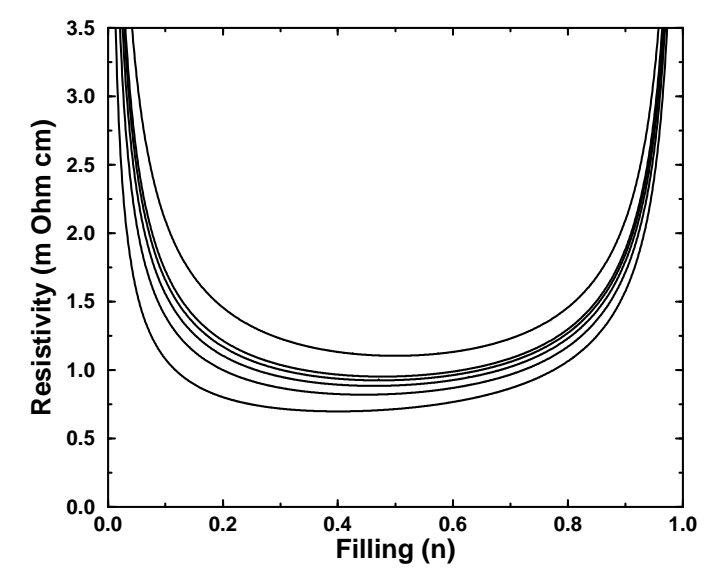

Figure 4. The zero field paramagnetic state resistivity $\rho$ (in $\mathrm{m} \Omega \mathrm{cm}$ ) versus filling $n$, plotted for $J=\infty, a=5 \AA$, and $S=1 / 2,1,3 / 2,2,5 / 2$, and $\infty, \rho$ increasing with $S$. The elliptical DOS and resistivity formula (51) are used.

\subsection{Lorentzian DOS}

In order to make contact with Furukawa's work [3] we now consider the effects of approximating the true cubic DOS $D_{\mathrm{c}}(\epsilon)$ with the Lorentzian $D_{\mathrm{l}}(\epsilon)$, which is somewhat less realistic than the elliptical DOS approximation owing to its slowly decaying tails. Our equations are very simple in this case: it may be shown that $J(\epsilon)=-i W$ and our equations for the Green function become explicit; switching on $W$ here merely broadens the peaks of the atomic limit spectral function into Lorentzians with the same width parameter $W$ as the bare DOS. Our approximation and Furukawa's become very similar in this case; in the classical $S \rightarrow \infty$ limit for example our local Green function is equal to Furukawa's.

The slowly decaying form of the Lorentzian means that the precise value of $J$ has more effect here than in the case of the elliptical DOS: the approximation $\langle\vec{S} \cdot \vec{\sigma}\rangle \approx n S / 2$ only holds accurately for very large $J$ and we only obtain a true insulator at half-filling for $J=\infty$ for instance. For simplicity however we take the limit $J \rightarrow \infty$ (with energy origin shifted by $-J S / 2$ ) rather than use the finite value used by Furukawa. In the 
paramagnetic state the local Green function $G$ is then

$$
G(\epsilon)=\frac{S+1-n / 2}{2 S+1} \frac{1}{\epsilon+i W}
$$

and the self-energy and chemical potential are

$$
\begin{aligned}
& \Sigma(\epsilon)=-\left(\frac{2 S+n}{2(S+1)-n}\right)(\epsilon+i W) \\
& \mu=W \tan \left[\frac{\pi}{2}\left(\frac{(4 S+3) n-2(S+1)}{2(S+1)-n}\right)\right] .
\end{aligned}
$$

If we use our finite-dimensional formula (51) to calculate $\rho$ with the Lorentzian DOS we must use a mixed approximation to the DOS, calculating $\phi$ using the Lorentzian approximation but using the elliptic approximation for $D_{\mathrm{c}}(\epsilon)$, in order for the integral in (51) to converge. This problem does not arise when using the infinite dimensional conductivity formula (53) so $D_{1}$ can be used throughout. The choice of conductivity formula (51) (with mixed approximation) or (53) does not qualitatively alter the results, but since the calculation using (51) has already been published [8] we use (53) here. It also seems more satisfactory to use the same approximation for the DOS throughout. We believe conductivity formula (51) to be more realistic however, as it does not rely on the infinite dimensions approximation.

$\rho$ is plotted against $n$ for various $S$ in figure 5. Note that the result is independent of $W$. As before we obtain insulating behaviour at $n=0$ and $n=1$, but now $\rho$ and $\partial \rho / \partial n$ are of the same order of magnitude as experiment for $n \sim 0.8$, and our results are also consistent with those of Furukawa. Even better agreement can be obtained by reducing $J$, which has the effect of reducing $\rho$ and $\partial \rho / \partial n$ for $n \sim 0.8$. Furukawa's result - that the DE model's prediction for $\rho$ is of the same order of magnitude as the experimental value - is therefore due to the unphysical approximation he used for the cubic DOS.

\section{Summary}

In addition to theoretical predictions of the properties of the DE model, experiments [6] also suggest that coupling to lattice degrees of freedom is a necessary ingredient in a model of CMR systems. However, we take the view that a good understanding of the bare DE model is necessary before more accurate and complicated models can be tackled. Accordingly in this paper we have presented a many-body CPA for the DE model and a compatible formula for the resistivity $\rho$; our main result is the reconciliation of the calculations of Millis et al [4] and Furukawa [3] of $\rho$ in the paramagnetic state. We have confirmed that single-site scattering within the DE model is inadequate to describe the resistivity of CMR systems.

Now the alloy CPA is known to give good results for a wide range of systems in which localization effects are unimportant. This should be the case here since, although some authors have claimed otherwise [20], localized states are expected to occur for the bare DE model only in the tails of the band; we therefore expect our formulae for $G_{\sigma}$ to be good approximations. Nevertheless several problems exist with our approximation. 


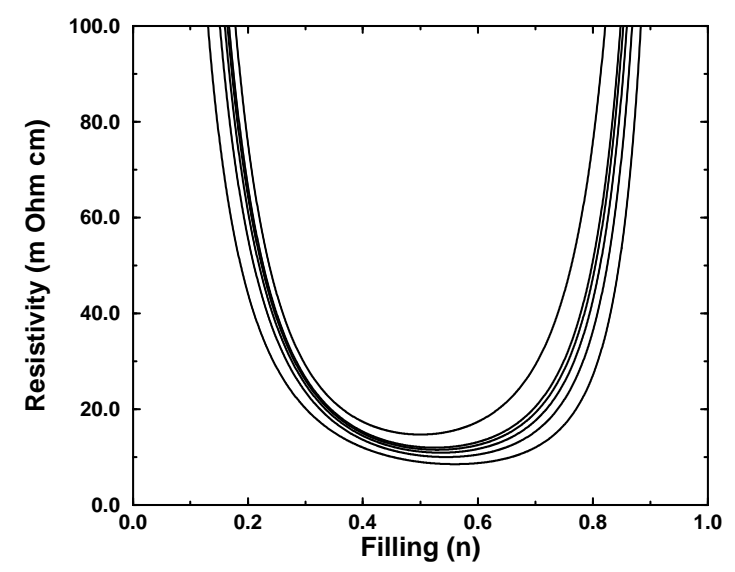

Figure 5. The zero field paramagnetic state resistivity $\rho$ (in $\mathrm{m} \Omega \mathrm{cm}$ ) versus filling $n$, plotted for $J=\infty, a=5 \AA$, and $S=1 / 2,1,3 / 2,2,5 / 2$, and $\infty, \rho$ increasing with $S$. The Lorentzian DOS and resistivity formula (53) are used.

Firstly, in common with the usual alloy CPA the imaginary part of the self-energy does not vanish at the Fermi level at $T=0$ so we do not obtain a true Fermi liquid; this should not be important in the high temperature paramagnetic regime that we have considered but the problem could perhaps be fixed following the approach of Edwards and Hertz to the Hubbard model [21]. Secondly, we have shown that for finite $S$ the saturated ferromagnetic state is never consistent in our approximation. Real CMR systems are believed to exhibit strong ferromagnetism in some regimes, and it is generally expected that the DE model has regions of strong ferromagnetism. Finally, although we recover Kubo's $n=0$ CPA in the low-density limit, we do not recover his strong-coupling $n=1$ CPA [12] at half-filling. This appears to be a shortcoming of our approximation, possibly due to our neglect of resonance broadening corrections. These problems and possible remedies will be discussed in a later paper in which we will concentrate on the magnetic susceptibility of our CPA.

\section{Acknowledgments}

KK was supported by EPSRC grant number GR/L90804 and JSPS grant number 09640453 and ACMG by an EPSRC studentship and a Monbusho REFYFR grant.

\section{References}

[1] Ramirez AP 1997 J. Phys.: Condens. Matter 9 8171-8199

[2] Zener C 1951 Phys. Rev. 82 403-405

[3] Furukawa N 1994 J. Phys. Soc. Japan 63 3214-3217

[4] Millis AJ, Littlewood PB and Shraiman BI 1995 Phys. Rev. Lett. 74 5144-5147

[5] Millis AJ, Shraiman BI and Mueller R 1996 Phys. Rev. Lett. 77 175-178 
[6] Zhao G, Conder K, Keller H and Müller KA 1996 Nature 381 676-678

[7] Edwards DM, Green ACM and Kubo K 1998 Physica B, In Press

[8] Kubo K, Edwards DM, Green ACM, Momoi T and Sakamoto H 1998 In Press

[9] Green ACM 1998 Correlated electrons in heavy fermion and double exchange systems (London: Imperial College $\mathrm{PhD}$ thesis)

[10] Hubbard J 1964 Proc. R. Soc. 281 401-419

[11] Fukuyama H and Ehrenreich H 1973 Phys. Rev. B 73266

[12] Kubo K 1972 J. Phys. Soc. Japan 33 929-935

[13] Kubo R 1957 J. Phys. Soc. Japan 12 570-586

[14] Kubo K 1974 J. Phys. Soc. Japan 36 32-38

[15] Müller-Hartmann E 1989 Z. Phys. B 74 507-512

[16] Brunton RE and Edwards DM 1998 J. Phys.: Condens. Matter 10 5421-5431

[17] Kubo K and Ohata N 1972 J. Phys. Soc. Japan 33 21-32

[18] Herrmann T and Nolting W 1996 Phys. Rev. B 53 10579-10588

[19] Urushibara A, Moritomo Y, Arima T, Asamitsu A, Kido G and Tokura Y 1995 Phys. Rev. B 51 14103-14109

[20] Varma CM 1996 Phys. Rev. B 54 7328-7333

[21] Edwards DM 1993 J. Phys.: Condens. Matter 5 161-170 